\title{
SEPARATION OF ARTERIAL PRESSURE INTO SOLITARY WAVES AND WINDKESSEL FLOW
}

\author{
Taous-Meriem Laleg* Emmanuelle Crépeau ** \\ Michel Sorine* \\ * INRIA Rocquencourt, 78153 Le Chesnay cedex, France \\ ** Laboratoire de mathématiques appliquées, UVSQ,
}

France

\begin{abstract}
A simplified model of arterial blood pressure intended for use in model-based signal processing applications is presented. The main idea is to decompose the pressure into two components: a travelling wave describes the fast propagation phenomena predominating during the systolic phase and a windkessel flow represents the slow phenomena during the diastolic phase. Instead of decomposing the blood pressure pulse into a linear superposition of forward and backward harmonic waves, as in the linear wave theory, a nonlinear superposition of travelling waves matched to a reduced physical model of the pressure, is proposed. Very satisfactory experimental results are obtained by using forward waves, the Nsoliton solutions of a Korteweg-de Vries equation in conjunction with a two-element windkessel model. The parameter identifiability in the practically important 3soliton case is also studied. The proposed approach is briefly compared with the linear one and its possible clinical relevance is discussed. Copyright (c)2006 IFAC
\end{abstract}

Keywords: pressure, wave, linear theory, nonlinear theory, identifiability

\section{INTRODUCTION}

In order to understand and assess the behaviour of the circulatory system both in normal and pathological conditions, many studies have been devoted to modelling the arterial tree. There are two types of models for arterial blood pressure (ABP) in large arteries. Lumped, or $0 D$, models ( $n D$ stands for $n$ space variables), like the popular windkessel models, are based on an analogy with simple RLC electrical circuits, the pressure being represented by a voltage and the blood flowrate by a current (Frank and Zhaorong, 1989). In order to enlarge its frequency domain, the basic two-element windkessel model, a resistance in parallel with a capacitor, has been extended to three and four elements by adding in series a resistance, alone or with an inductance in parallel (Stergiopulos et al., 1999). Windkessel models successfully explain the diastolic phase but, with their low order, they can not explain propagation phenomena like the transit delay of the pressure pulse. The $3 D$ distributed models are based on computational fluid dynamic principles and can explain observed phenomena (McDonald, 1974). Too complex for some applications, they can be reduced to $2 D$ or $1 D$ models (Canic et al., 2005). Then $0 D$ models can be deduced from $1 D$ models (Monti et al., 2002) and (Milisic and Quarteroni, 2004 ) to be used as boundary conditions for $1 D$ models (Olufsen, 1999) and (Fernandez et al., 2005) or in signal analysis as is done here.

During the propagation of the Pressure Pulse (PP) along the arterial tree, phenomena like "Peaking" and "Steepening" are observed. This has been explained by the linear superposition of direct and reflected waves, the reflected waves 
being created when the forward waves, from the heart to the periphery, encounter discontinuities in the arterial properties like a bifurcation or a stenosis. This model has been well known for decades and many studies have been carried out in order to separate the PP into its forward and backward components, as in the pioneering work of Westerhof (Westerhof et al., 1972), followed by many others (Li, 1986), (Parker and Jones, 1990), (Berger et al., 1993), (Stergiopulos et al., 1993), (Pythoud et al., 1995) and (Pythoud et al., 1996).

The main idea in this article is to decompose the pressure into a travelling wave representing the fast propagation phenomena during the systolic phase and a windkessel term representing slow phenomena during the diastolic phase. A decomposition of this kind has been studied in (Wang et al., 2002) but the novelty here consists in choosing a forward solitary wave which already captures essential properties of the $\mathrm{PP}$, like the "Peaking" and the "Steepening" phenomena. Solitons resulting from a balance between shock wave creation and wave dispersion are proposed. They possess an analytical expression which leads to a reduced ABP model that is easy to identify, an important advantage of the approach. So, instead of the usual decomposition of the ABP as a linear superposition of forward and backward harmonic waves, the suggestion is to use a nonlinear superposition of forward solitary waves completed by a windkessel flow. The use of solitons for analyzing the ABP has been proposed in (Crépeau and Sorine, 2005), where based on results of (Yomosa, 1987) a quasi-1D Navier-Stokes equation is chosen and reduced to a Korteweg-de Vries equation (KdVE) with solitons as particular solutions. In (Crépeau and Sorine, 2005), it is shown that 2 or 3 interacting solitons are sufficient for a good description of the systolic phase.

The next section, after recalling the linear theory, presents a soliton + windkessel correction-term decomposition needed in the diastolic phase. In section 3 , the identifiability of the 3 -soliton's parameters and of a two-element windkessel model is studied. In section 4 experimental results are presented, followed by a discussion in section 5 .

\section{REDUCED ARTERIAL BLOOD PRESSURE MODEL}

The blood pressure $P(z, t)$ and flow $Q(z, t)$, with $z$ the position on the arterial tree and $t$ the time, are described in (Westerhof et al., 1972) as the following linear superposition of forward and backward waves (with subscripts $f$ and $b$ respectively):

$$
P(z, t)=P_{f}\left(z-c_{0} t\right)+P_{b}\left(z+c_{0} t\right)
$$

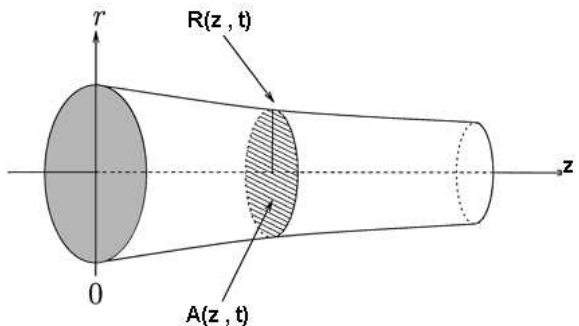

Fig. 1. Blood flow vessel

$$
Q(z, t)=Q_{f}\left(z-c_{0} t\right)+Q_{b}\left(z+c_{0} t\right),
$$

$c_{0}=\sqrt{\frac{E h_{0}}{2 \rho R_{0}}}$ is the Moens-Korteweg sound waves velocity in an elastic vessel filled with blood of density $\rho . E$ is the wall elasticity coefficient. $R_{0}$ and $h_{0}$ are respectively the equilibrium radius and the thickness of the vessel. The impedance $Z(z, \omega)$ at position $z$ and frequency $\omega$ is given by the ratio of the time Fourier transforms of the pressure and flow at this position:

$$
Z(z, \omega)=\frac{\hat{P}(z, \omega)}{\hat{Q}(z, \omega)}=\frac{\hat{P}_{f}(z, \omega)+\hat{P}_{b}(z, \omega)}{\hat{Q}_{f}(z, \omega)+\hat{Q}_{b}(z, \omega)} .
$$

The global reflection coefficient $\Gamma$ is given by (Berger et al., 1993) and (Westerhof et al., 1972):

$$
\Gamma(z, \omega)=\frac{Z(z, \omega)-Z_{c}}{Z(z, \omega)+Z_{c}} .
$$

where $Z_{c}$ denotes the characteristic impedance that can be estimated by several methods (in the case of a reflectionless tube $\left.Z_{c}=Z\right)(\mathrm{Li}, 1982),(\mathrm{Li}$, 1986), (Quick et al., 2001) and (Westerhof et al., 1972). Knowing $Z$ and $Z_{c}$ it is possible to estimate $\Gamma$ and the forward and backward components of the pressure are given by (Westerhof et al., 1972) and (Westerhof et al., 2005):

$$
\begin{aligned}
\hat{P}_{f}=\frac{\hat{P}}{1+\Gamma}, & \hat{Q}_{f}=\frac{\hat{Q}}{1-\Gamma}, \\
\hat{P}_{b}=\Gamma \hat{P}_{f}, & \hat{Q}_{b}=-\Gamma \hat{Q}_{f} .
\end{aligned}
$$

This method has the advantage of requiring pressure and flow measurements at only one location but it assumes a linear model of the flow, the impedance. Many studies have followed, aiming to take the nonlinearities into account when separating the PP into forward and backward components. In (Parker and Jones, 1990) and (Stergiopulos et al., 1993), quasi-nonlinear methods for $1 D$ flow models are used (method of characteristics and split coefficient matrix for the Euler equation) under the linearizing assumption that intersecting forward and backward waves 
are additive. In (Berger et al., 1993) the linear superposition of multiple forward and reflected waves is considered. In (Pythoud et al., 1995) and (Pythoud et al., 1996), a nonlinear separation is proposed using Riemann invariants which takes into account nonlinearities in the area-pressure relationship and in the convective term.

Surprisingly, if the radial acceleration is also taken into account in a quasi $1 D$ Navier-Stokes equation, the model becomes simpler as observed in (Crépeau and Sorine, 2005): using a singular perturbation technique, it leads to a KdVE, a simpler situation because this equation is integrable, admitting soliton solutions. As proposed in (Crépeau and Sorine, 2005), solitons are then a natural choice of base functions to analyze the ABP, the reference model being the KdVE:

$$
\frac{\partial P_{s}}{\partial z}+\left(d_{0}+d_{1} P_{s}\right) \frac{\partial P_{s}}{\partial t}+d_{2} \frac{\partial^{3} P_{s}}{\partial t^{3}}=0,
$$

where $P_{s}(z, t)$ is the $\mathrm{ABP}$ and

$$
d_{0}=\frac{1}{c_{0}}, d_{1}=-\frac{2 \alpha+1}{2 \rho c_{0}^{3}}, d_{2}=-\frac{\rho_{\omega} h_{0} R_{0}}{2 \rho c_{0}^{3}}
$$

with $\alpha$ a momentum-flux correction coefficient and $\rho_{\omega}$ the wall density. Remark in $(7)$ the pressure dependent velocity $d_{0}+d_{1} P_{s}$ and the dispersion term $\frac{\partial^{3} P_{s}}{\partial t^{3}}$ that are at the origin of solitons.

With the new variables $\xi=t-d_{0} z, \tau=d_{2} z$ and $y=\frac{d_{1}}{6 d_{2}} P_{s},(7)$ becomes a normalized KdVE:

$$
\frac{\partial y}{\partial \tau}+6 y \frac{\partial y}{\partial \xi}+\frac{\partial^{3} y}{\partial \xi^{3}}=0, \quad y(\xi, 0)=y_{0}(\xi) .
$$

The general analytical expression of an N-soliton solution of (8) can be found in (Whitham, 1999):

$$
y(\xi, \tau)=2 \frac{\partial^{2}(\ln \operatorname{det}(\mathbf{M}))}{\partial \xi^{2}},
$$

M is a $N \times N$ matrix with coefficients given by:

$$
M_{m k}=\delta_{m k}+\frac{2 a_{m}}{a_{m}+a_{k}} f_{m}, \quad m, k=1 \ldots N
$$

where $\delta_{m k}$ is the Kronecker symbol and

$$
\begin{aligned}
& f_{m}(\xi, \tau)=\exp \left[-a_{m}\left(\xi-s_{m}-a_{m}^{2} \tau\right)\right] \\
&\left(a_{m}, s_{m}\right) \in \mathbb{R}^{+} \times \mathbb{R} .
\end{aligned}
$$

One can notice from real data that the ABP can be approximated by 2 or 3 -solitons. In the following, 3-solitons are considered. The parameters $d_{0}$, $d_{1}$ and $d_{2}$ characterize the KdVE and depend on the characteristics of the vascular compartment while the parameters $a_{1}, a_{2}$ and $a_{3}$ characterize the solitons. As is noticed in (Crépeau and Sorine, 2005) solitons lead to a good estimation of the systolic phase but need some correction in the diastolic phase. So this work proposes to describe slow phenomena with a two-element windkessel model. Then, the ABP can be written in the following form:

$$
P(z, t)=\bigoplus_{j=1}^{n}\left(P_{s j}(z, t)\right)+P_{w k}(t),
$$

where $\bigoplus_{j=1}^{n} P_{s j}(z, t)$ is a wave term given by a nonlinear superposition of solitary waves $P_{s j}(z, t)$. For example a 2-soliton can be written as a nonlinear superposition of two solitary waves. $P_{w k}(t)$ is the output of a two-element windkessel model given by the following differential equation (Stergiopulos et al., 1999) and (Wang et al., 2002):

$$
\frac{d P_{w k}}{d t}(t)+\frac{P_{w k}(t)}{R C}=\frac{P_{\infty}}{R C}+\frac{Q(t)}{C},
$$

where $R$ denotes the peripheral resistance, $C=$ $\frac{d V_{w k}}{d P_{w k}}$ the compliance of the arteries and $Q(t)$ the inflow. The inflow can be taken proportional to $P_{s}$ (Wang et al., 2002). So it is written $Q=\frac{P_{s}}{R_{s}}$.

\section{IDENTIFIABILITY OF THE MODEL'S PARAMETERS}

\subsection{Identifiability of a 3-soliton's parameters}

In this section identifiability of the 3-soliton's parameters is studied. For this purpose, the normalized KdVE (8) is considered. Then, a 3-soliton solution of (8) is given by (9). For studying identifiability, the definition given in (Walter and Pronzato, 1994) is used. So, two solutions of (8) in the form of a 3-soliton are assumed such that:

$$
M_{m k}^{j}=\delta_{m k}+\frac{2 a_{m}^{j}}{a_{m}^{j}+a_{k}^{j}} f_{m}^{j},
$$

where $j=1,2, m=1,2,3$ and $k=1,2,3$.

The determinant of $\mathbf{M}^{j}, j=1,2$ is given by:

$$
\begin{array}{r}
\operatorname{det}\left(\mathbf{M}^{j}\right)=1+f_{1}^{j}+f_{2}^{j}+f_{3}^{j}+\left(\frac{a_{1}^{j}-a_{2}^{j}}{a_{1}^{j}+a_{2}^{j}}\right)^{2} f_{1}^{j} f_{2}^{j}+ \\
\left(\frac{a_{1}^{j}-a_{3}^{j}}{a_{1}^{j}+a_{3}^{j}}\right)^{2} f_{1}^{j} f_{3}^{j}+\left(\frac{a_{2}^{j}-a_{3}^{j}}{a_{2}^{j}+a_{3}^{j}}\right)^{2} f_{2}^{j} f_{3}^{j}+ \\
\left(1+\frac{16 a_{1}^{j} a_{2}^{j} a_{3}^{j}}{\left(a_{1}^{j}+a_{2}^{j}\right)\left(a_{1}^{j}+a_{3}^{j}\right)\left(a_{2}^{j}+a_{3}^{j}\right)}-\frac{4 a_{1}^{j} a_{2}^{j}}{\left(a_{1}^{j}+a_{2}^{j}\right)^{2}}-\right. \\
\left.\frac{4 a_{1}^{j} a_{3}^{j}}{\left(a_{1}^{j}+a_{3}^{j}\right)^{2}}-\frac{4 a_{2}^{j} a_{3}^{j}}{\left(a_{2}^{j}+a_{3}^{j}\right)^{2}}\right) f_{1}^{j} f_{2}^{j} f_{3}^{j} .
\end{array}
$$

For $\tau=0, f_{i}^{j}$ can be rewritten as follows:

$$
f_{i}^{j}(\xi, 0)=\exp \left(-a_{i}^{j} \xi\right) \exp \left(a_{i}^{j} s_{i}^{j}\right),
$$

where $j=1,2$ and $i=1,2,3$. 
The $a_{i}^{j}$ are positive and can be ordered: $a_{1}^{j}>$ $a_{2}^{j}>a_{3}^{j}$. Then when $\xi \rightarrow+\infty$, the asymptotic behaviour is:

$\operatorname{det}\left(\mathbf{M}^{j}\right)=k^{j} \exp \left(-\left(a_{1}^{j}+a_{2}^{j}+a_{3}^{j}\right) \xi\right), j=1,2 .(15)$

Now, the question is: does $y^{1}(\xi, 0)=y^{2}(\xi, 0)$ imply unicity of the 3 -soliton (i.e $\mathbf{M}^{1}=\mathbf{M}^{2}$ )? When $y^{1}(\xi, \tau)=y^{2}(\xi, \tau)$ :

$$
\frac{\partial^{2}\left(\ln \operatorname{det}\left(\mathbf{M}^{1}\right)\right)}{\partial \xi^{2}}=\frac{\partial^{2}\left(\ln \operatorname{det}\left(\mathbf{M}^{2}\right)\right)}{\partial \xi^{2}} .
$$

Remark that equation (9) with the boundary condition (15) is a Poisson equation. Therefore, from the Dirichlet theorem, its solution is unique. So,

$$
\operatorname{det}\left(\mathbf{M}^{1}\right)=\operatorname{det}\left(\mathbf{M}^{2}\right) .
$$

Now, does the unicity of the determinant of such a matrix (13) imply the unicity of the matrix and therefore the unicity of the 3-soliton? From the asymptotic behaviour (15):

$$
\begin{gathered}
\text { if } \quad \operatorname{det}\left(\mathbf{M}^{1}\right)=\operatorname{det}\left(\mathbf{M}^{2}\right), \\
\text { then } \quad a_{1}^{1}+a_{2}^{1}+a_{3}^{1}=a_{1}^{2}+a_{2}^{2}+a_{3}^{2} .
\end{gathered}
$$

By isolating the different exponential terms in the determinant expression, different cases have to be studied. For example, $a_{1}^{2}+a_{2}^{2}$ is equal to either $a_{1}^{1}+a_{2}^{1}$ or $a_{2}^{1}+a_{3}^{1}$ or $a_{1}^{1}+a_{3}^{1}$. So the solutions $a_{j}^{2}$ consist of a permutation of the $a_{j}^{1}$. A similar result is found for the $s_{j}^{2}$. So, there exists a finite number of matrices $\mathbf{M}$ such that: $\operatorname{det}\left(\mathbf{M}^{2}\right)=\operatorname{det}\left(\mathbf{M}^{1}\right)$. But, it is important to notice that the greatest of the $a_{j}^{2}$ coincides with the greatest of $a_{j}^{1}$ and so on. Therefore, if $a_{1}^{2}>a_{2}^{2}>a_{3}^{2}$ then the following unique solution is found:

$$
a_{i}^{2}=a_{i}^{1}, \quad s_{i}^{2}=s_{i}^{1}, \quad i=1,2,3 .
$$

In other terms, if $\operatorname{det}\left(\mathbf{M}^{1}\right)=\operatorname{det}\left(\mathbf{M}^{2}\right)$ then the 3 -solitons defined by $\mathbf{M}^{1}$ and $\mathbf{M}^{2}$ are the same.

\subsection{Identifiability of the two-element windkessel model}

Assuming that $P_{s}(t)$ is known, the parameters to identify are $R, C, P_{\infty}$ and $R_{s}$ in equation (12). Let $P_{w k}^{j}(t), j=1,2$ be such that:

$$
P_{w k}^{1}(t)=P_{w k}^{2}(t)
$$

The equality of the corresponding parameters, $R^{j}, C^{j}, P_{\infty}^{j}$ and $R_{s}^{j}, j=1,2$ has to be shown. Applying the Laplace transform to equation (12) gives:

$$
\left(s+\frac{1}{R C}\right) P_{w k}(s)-P_{0}=\frac{1}{s} \frac{P_{\infty}}{R C}+\frac{P_{s}(s)}{R_{s} C},
$$

then, for $j=1,2, P_{w k}^{j}$ is given by:

$$
P_{w k}^{j}(s)=\frac{s R^{j} C^{j} P_{0}^{j}+P_{\infty}^{j}}{s\left(1+s R^{j} C^{j}\right)}+\frac{\frac{R^{j}}{R_{s}^{j}} P_{s}(s)}{1+s R^{j} C^{j}} .
$$

From equations (19) and (21) it can be deduced:

- For the pole 0: $P_{\infty}^{1}=P_{\infty}^{2}$.

- For the zero at infinity :

$$
P_{0}^{1}+\frac{1}{R_{s}^{1} C^{1}} \lim _{s \rightarrow \infty} P_{s}(s)=P_{0}^{2}+\frac{1}{R_{s}^{2} C^{2}} \lim _{s \rightarrow \infty} P_{s}(s) .
$$

As

$$
P_{s}(0)=\lim _{s \rightarrow \infty} s P_{s}(s)<+\infty,
$$

it follows:

$$
P_{0}^{1}=P_{0}^{2} \quad \text { and } \quad R_{s}^{1} C^{1}=R_{s}^{2} C^{2} .
$$

- For the finite pole: $R^{1} C^{1}=R^{2} C^{2}$.

It can be noticed that the parameters $R, C$ and $R_{s}$ are not identifiable. However $T=R C, T_{s}=R_{s} C$ and $P_{\infty}$ are identifiable, so that it is better to write equation (12) as follows:

$$
\frac{d P_{w k}}{d t}(t)+\frac{P_{w k}(t)}{T}=\frac{P_{\infty}}{T}+\frac{P_{s}(t)}{T_{s}} .
$$

The parameters to identify are now $T, P_{\infty}$ and $T_{s}$.

\section{EXPERIMENTAL RESULTS}

Some first comparisons of measured and computed ABP signals are presented. ABP has been measured at the finger with a FINAPRES. The parameters of the two parts of our model, the 3soliton and the windkessel flow, were estimated from real data. Figures 2 and 3 illustrate the satisfactory experimental results obtained. First, the model was superposed to a single beat. Then the procedure was extended to a sequence of beats. In figure 4 the estimation error when using a 3soliton alone is represented and is well approximated by a two-element windkessel model.

\section{DISCUSSION}

The different phenomena observed when the PP propagates along the arterial tree were explained in the linear theory by the existence of backward waves. For example, the "Peaking" was associated with the increase of the backward wave velocity, this wave going back earlier. The increase in the velocity results from the changes in the vessels' characteristics (the increase in stiffness and the decrease in section). However, the nonlinear superposition of forward solitons can also explain this phenomenon. Therefore, the "Peaking" can be explained by the increase in the soliton velocity 
which leads to the increase in its amplitude (this is one of the interesting characteristics of the solitons). The "Steepening" also can be explained by the conservation laws: the increase in the amplitude leads to a decrease in the width.

There is always some arbitrariness in choosing a function basis to represent the solution of an evolution equation, unless this basis has some special properties as in the case of eigenfunctions for a linear system. The decomposition of the PP wave into a superposition of harmonic forward and backward waves corresponds to a small amplitude, high frequency approximation of the linearized flow equations which, in this case can be reduced into a linear second order wave equation. It is also a convenient representation of functions due to Fourier analysis and calculus. This linear decomposition of waves necessitates in general between 6 and 12 components. As described before, the proposed approach, due to the choice of forward waves matched to the pressure waveform (in fact particular solutions of a reduced model of the flow) can neglect the backward waves and needs only a small number of components: 2 or 3 solitons are sufficient. An important advantage of this approach is that it leads to a reduced PP model with a small number of identifiable parameters, as was proved in the previous section.

The linear theory leads to a good local model of the PP (Westerhof et al., 1972) but it can not help to solve the problem of the distal-to-proximal transfer function estimation. Remark that this interesting problem is still open (Remmen et al., 2002). It gives a good description of the PP at the measurement point of the arterial tree but it doesn't allow the description of the PP in a different location because it does not take into account propagation phenomena. The nonlinear superposition of solitons constitutes a global model in time and space and it is a good candidate to tackle this problem of transfer-function estimation. This may lead to the estimation of proximal pressure (at aortic or ventricle levels) from distal one (at finger level for example) using only non-invasive measurements. This problem is the subject of current research.

Another important point concerns clinical applications. In the case of the linear decomposition, pressure and flow measurements at a same point are needed. But, it is difficult to have joint measurements of flow-rate and aortic pressure. The latter can be obtained with invasive techniques and the former from image processing. In the proposed approach, only non-invasive pressure measurements are needed to identify the model. This is potentially an important advantage. Also, with this approach, some parameters are identified which have to be interpreted. The arterial tree is represented by the characteristics $d_{i}, i=0,1,2$ of an equivalent unique vessel and of a windkessel model; the PP by the parameters $a_{j}, j=1,2,3$ of a soliton. All these parameters seem to give new insight on the blood flow.

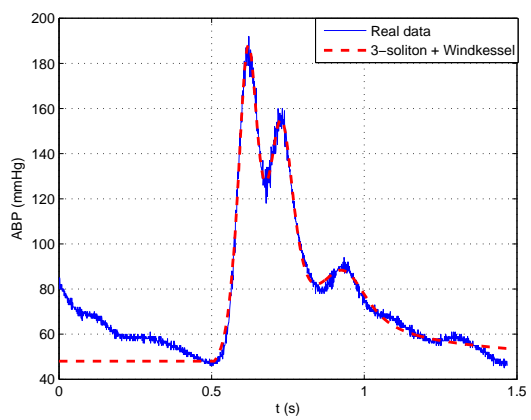

Fig. 2. Pressure at the finger: real and estimated data

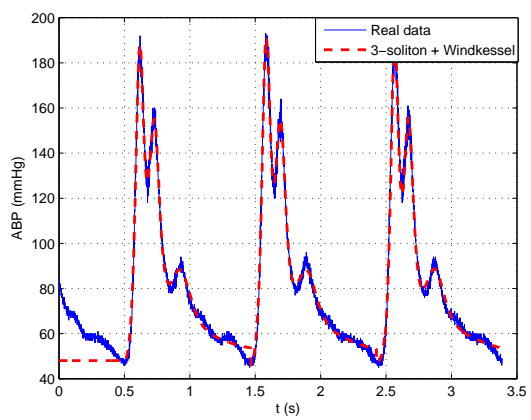

Fig. 3. Pressure at the finger: real and estimated data

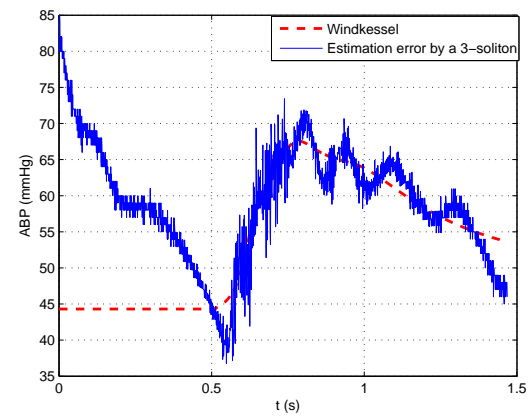

Fig. 4. The estimation error by a 3-soliton alone is well approximated by a two-element windkessel model

\section{CONCLUSION}

In this paper, a reduced blood pressure model is proposed. The theoretical considerations suggest 
that ABP can be seen as the sum of two terms: an $\mathrm{N}$-soliton (with $\mathrm{N}=2$ or 3 in the present experiments) and a two-element windekessel model. The former takes into account fast wave phenomena and the latter slow phenomena. The agreement between estimated and real pressure is satisfactory. The introduction of forward solitons explains the "Peaking" and the "Steepening" phenomena. Unlike the linear approach, which necessitates simultaneous blood pressure and flow measurements, the proposed model requires only pressure measurements. It depends on a small number of parameters that are easy to identify. It seems that these results on ABP waveform analysis, in particular in the systolic phase, can lead to some interesting clinical applications.

\section{ACKNOWLEDGMENTS}

The authors would like to thank Y.Papelier and Hospital Beclere for providing us pressure data.

\section{REFERENCES}

Berger, D.S., J.K.J. Li, W.K. Laskey and A. Noordergraaf (1993). Repeated reflection of waves in the systemic arterial system. The American Physiological Society pp. 269-281.

Canic, S., D. Lamponi, A. Mikelic and J. Tambaca (2005). Self-consistent effective equations modeling blood flow in medium-to-large compliant arteries. SIAM Multiscale Modeling and Simulation 3(3), 559-596.

Crépeau, E. and M. Sorine (2005). Identifiability of a reduced model of pulsatile flow in an arterial compartment. IEEE CDC and ECC.

Fernandez, M., V.Milisic and A. Quarteroni (2005). Analysis of a geometrical multiscale blood flow model based on the coupling of ode's and hyperbolic pde's. SIAM Multiscale Modeling and Simulation 4(1), 215-236.

Frank, C.P. and L. Zhaorong (1989). Estimating arterial resistance and compliance during transient conditions in humans. Am. J. Physiol (257), 190-197.

Li, J.K.J. (1982). Cardiovascular diagnostic parameters derived from pressure and flow pulses. IEEE Frontiers of Engineering in Health Care pp. 531-534.

Li, J.K.J. (1986). Time domain resolution of forward and reflected waves in the aorta. IEEE Transactions on Biomedical Engineering 33(8), 783-785.

McDonald, D.A. (1974). Blood flow in arteries. 2nd ed.. Edward Arnold.

Milisic, V. and A. Quarteroni (2004). Analysis of lumped parameter models for blood flow simulations and their relation with $1 \mathrm{~d}$ models. M2AN IV, 613-632.
Monti, A., C. Medigue and M. Sorine (2002). Short-term modelling of the controlled cardivascular system. In: ESAIM:Proceedings. Vol. 12.

Olufsen, M. (1999). structured tree outflow condition of blood flow in larger systemic arteries. The American Physiological Society.

Parker, K.H. and J.H. Jones (1990). Forward and backward running waves in arteries: analysis using the method of characteristics. ASME J. Biomech. Eng (112), 322-326.

Pythoud, F., N. Stergiopulos and J.J. Meister (1995). Forward and backward waves in the arterial system: nonlinear separation using riemann invariants. Technology and Health Care 3, 201-207.

Pythoud, F., N. Stergiopulos and J.J. Meister (1996). Separation of arterial pressure waves into their forward and backward running components. Journal of Biomechanical Engineering 118, 295-301.

Quick, C.M., D.S. Berger and A. Noordergraaf (2001). Constructive and destructive addition of forward and reflected arterial pulse waves. AJP-Heart and Circulatory Pysiology (280), 1519-1527.

Remmen, J.J., W.R. Aengevaeren and F.W. Verheugt et al (2002). Finapres arterial pulse wave analysis with modelflow is not a reliable non-invasive method for assessment of cardiac output.. Clinical Science (103), 143-149.

Stergiopulos, N., B.E. Westerhof and N. Westerhof (1999). Total arterial inertance as the fourth element of the windkessel model. The American Physiological Society pp. 81-88.

Stergiopulos, N., N. Tardy and Y. Meister (1993). Nonlinear separation of forward and backward running waves in elastic conduits. J.Biomechanics (26), 201-209.

Walter, E. and L. Pronzato (1994). Identification de modèles paramétriques à partir de données expérimentales. Edition Masson.

Wang, J.J., A.B. O'Brien, N.G. Shrive, K.H. Parker and J.V. Tyberg (2002). Time-domain representation of ventricular-arterial coupling as a windkessel and wave system. AM J Physiol Heart Circ Physiol.

Westerhof, N., N. Stergiopulos and M.I.M. Noble (2005). Snapshots of Hemodynamics, An Aid for Clinical Research and Graduate Education. Springer Science.

Westerhof, N., P. Sipkema, G.C. VanDenBos and G. Elzinga (1972). Forward and backward waves in the arterial system. Cardiovascular Research 6, 648-656.

Whitham, G.B. (1999). Linear and nonlinear Waves. J.Wiley \& sons.

Yomosa, S. (1987). Solitary waves in large vessels. Journal of the Physical Society of Japan 50(2), 506-520. 